\title{
Immunotherapy in oncogene addicted non-small cell lung cancer
}

\author{
Luke McLean $^{1 \#}$, Jose Luis Leal ${ }^{1 \#}$, Benjamin J. Solomon ${ }^{1,2}$, Thomas John ${ }^{1,2}$ \\ ${ }^{1}$ Department of Medical Oncology, Peter MacCallum Cancer Centre, Melbourne, Victoria, Australia; ${ }^{2}$ Sir Peter MacCallum Department of \\ Oncology, University of Melbourne, Parkville, Victoria, Australia \\ Contributions: (I) Conception and design: All authors; (II) Administrative support: All authors; (III) Provision of study materials or patients: All \\ authors; (IV) Collection and assembly of data: All authors; (V) Data analysis and interpretation: All authors; (VI) Manuscript writing: All authors; (VII) \\ Final approval of manuscript: All authors. \\ \#These authors contributed equally to this work. \\ Correspondence to: Associate Professor Thomas John, MBBS, PhD, FRACP. Department of Medical Oncology, Peter MacCallum Cancer Centre, 305 \\ Grattan Street, Melbourne 3000, Victoria, Australia. Email: tom.john@petermac.org.
}

\begin{abstract}
The use of immune checkpoint inhibitors (ICIs) targeting the programmed cell death-1 (PD-1) and programmed cell death ligand-1 (PD-L1) has led to notable changes in treatment strategies for patients with advanced non-small cell lung cancer (NSCLC) and now forms a part of standard of care treatment in patients with advanced disease. However, most patients do not respond to ICI monotherapy, which may be explained by significant variations in efficacy according to different immune and molecular profiles in tumours. Improved response rates have been observed in smokers and are associated with tumors that have high mutation loads, with a higher tendency to form neoantigens. This premise itself defies the eventual significance of ICIs for oncogene-driven NSCLC, which in general are more common in never smokers and potentially have reduced capacity for neoantigen formation. Furthermore, pivotal trials investigating ICIs in advanced NSCLC have usually excluded patients with oncogenic drivers, hence the outcome of these agents in this population is poorly characterized. In this article, we aim to review the most current evidence, encompassing clinical and preclinical data focused on a wide range of oncogene-addicted NSCLCs.
\end{abstract}

Keywords: Oncogene; immunotherapy; lung cancer; tyrosine kinase inhibitors (TKIs)

Submitted Jun 22, 2020. Accepted for publication Aug 10, 2020.

doi: $10.21037 /$ tlcr-20-772

View this article at: http://dx.doi.org/10.21037/tlcr-20-772

\section{Introduction}

Lung cancer is the leading cause of cancer-related mortality worldwide and non-small cell lung cancer (NSCLC) accounts for almost $85 \%$ of all lung cancer cases (1). In the last decade the identification of various oncogenes (see Figure 1), development of targeted therapies and more recently immunotherapies have refined and indeed transformed therapeutic algorithms for NSCLC. Genotypedirected treatments targeting oncogene-addicted NSCLC, including EGFR (5-8) and BRAF V600E mutations (9) or ALK (10-12), ROS1 (13) and RET (14) oncogenic rearrangements, have demonstrated high response rates and prolonged survival resulting in their adoption as front line therapies. By contrast, immunotherapies when used as single agents are ineffective in the majority of patients, although offer long term benefits in some patient subgroups-particularly when used in combinations with chemotherapy.

While the efficacy of targeted therapies is contingent on the target being detectable within the tumour, predictive markers for immunotherapy are less reliable. Several studies have indicated that PD-L1 expression, tumour-infiltrating lymphocytes (TILs), tumour mutational burden (TMB), neoantigens and DNA mismatch repair (MMR) deficiency may serve as potential predictive biomarkers for immune checkpoint inhibitor (ICI) effectiveness. The main biomarker that has emerged as clinically useful is tumour cell PDL1 expression as assessed by immunohistochemistry. 


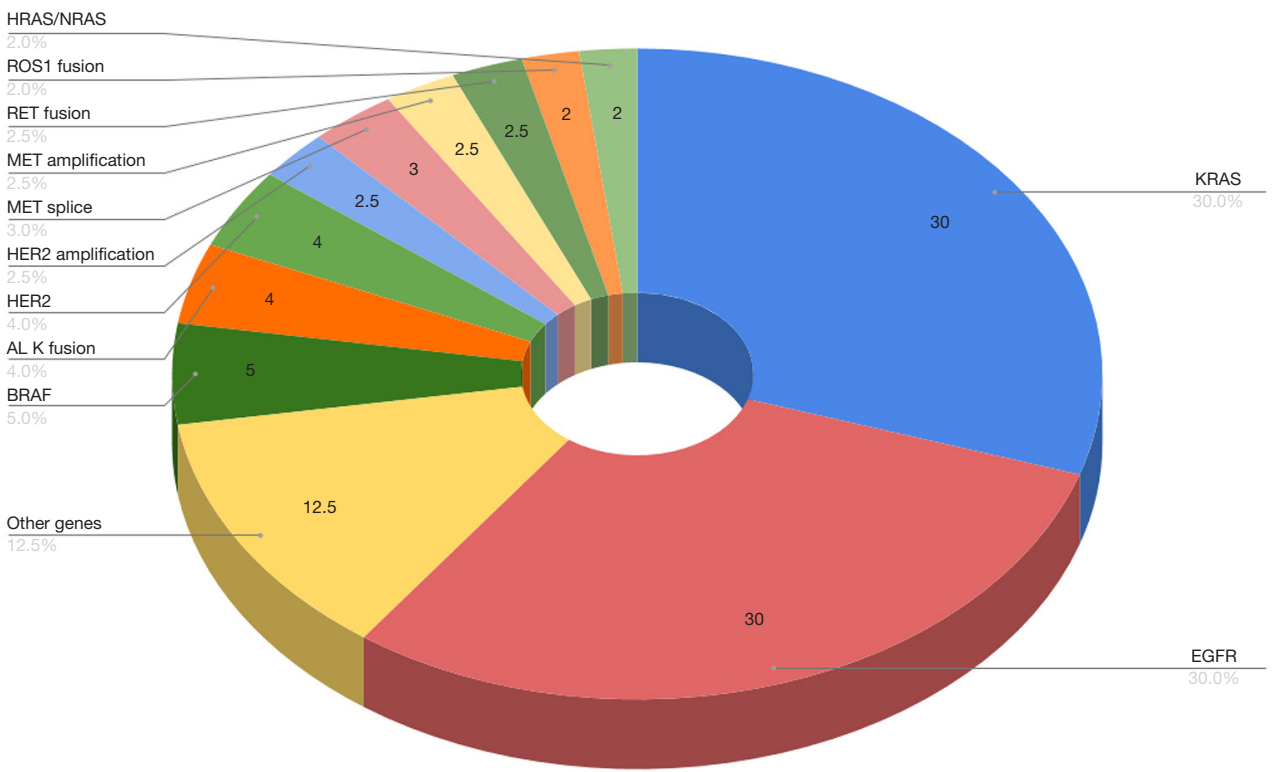

Figure 1 Single oncogenic drivers in metastatic lung adenocarcinoma. Oncogenic driver alterations in advanced NSCLC (including treatment-naive patients and patients who had previously received anticancer therapies). Data adapted from Skoulidis et al. (2), based on next-generation sequencing of predefined panels from patients treated at the Memorial Sloan Kettering Cancer Center [n=860; MSKIMPACT panel (Jordan et al.) (3)] and samples referred to Foundation Medicine [n=4,402; FoundationOne panel (Frampton et al.) (4)] ( $\mathrm{n}=5,262$ patients with advanced/metastatic NSCLC in total). The increased prevalence of EGFR mutations in the metastatic dataset may partially reflect referral bias.

However, even in patients with high expression of PDL1, about $50 \%$ of patients do not respond to single agent ICI and some patients negative for PD-L1 experience strong and durable responses. Such diversity of response cannot be purely explained by tumour heterogeneity suggesting that other factors, either within the tumour, the host, or both must be at play. Oncogene addicted tumours collectively represent a broad set of tumours and the role of immunotherapy from existing trials may not be broadly generalizable. Phase three data in this setting for oncogene addicted tumours is unfortunately limited, given that many trials excluded these patients, and when included they represent small subsets depending on the type of oncogenic driver.

In this article, we review the clinical data available regarding outcome of oncogene addicted NSCLC patients treated with ICIs and the primary preclinical evidence concerning immunologic characteristics in oncogenedriven NSCLC, which seems to be critical in unravelling the potential benefit of these agents in this specific patient population.

\section{Epidermal growth factor receptor (EGFR) mutations}

\section{Current practice and the limitations of TKIs}

Actionable EGFR mutations vary in their incidence from $15 \%$ up to $65 \%$ of lung adenocarcinomas with the higher incidence occurring in East Asian populations (15) and are associated with improved response rates to tyrosine kinase inhibitor (TKI) therapy (16). Most treatment algorithms mandate initial testing for EGFR to guide first line therapy given the efficacy and low toxicity of TKIs compared to chemotherapy (17-23). Inevitably, however, resistance to TKIs develops and chemotherapy is currently the main subsequent treatment employed after failure of TKI options.

\section{Rationale for immunotherapy}

The interest in immunotherapy was supported by preclinical studies demonstrating that PD-L1 expression by EGFR-mutant tumours increased as a mechanism of immune evasion (24) and that PD-L1 overexpression by 
tumour cells occurred through the ERK1/2-c-jun pathway by activating mutant EGFR (25). Discordant treatment effects have however been seen in vitro with erlotinib reducing PD-L1 expression in one study (26) and gefitinib increasing PD-L1 in serial biopsies in a clinical setting (27). To add to a lack of consistency, Gainor and colleagues found in their retrospective analysis that PD-L1 expression was low prior to TKI exposure (16\%) and at acquired resistance (29\%) (28). Similarly, Offin and colleagues reported TMB increased with resistance, but that the overall levels were still low (29). Whilst the presence of an actionable EGFR mutation is associated with increased TKI response rates there is a lack of a robust marker for immunotherapy agents (30) and PD-L1 and TMB may not be informative in this context.

Further evidence from a retrospective series has also suggested EGFR TKI resistance results in increased TMB which has supported the current practice of sequencing TKIs prior to immunotherapy (29). The lack of benefit from immunotherapy has been hypothesised to be due to several factors. Pre-clinical studies suggest a "cold" and more immunosuppressive tumour microenvironment with reduced tumour infiltrating lymphocytes and $\mathrm{T}$ cell infiltration exists in EGFR-mutant lung cancers $(31,32)$ as well as CD73 overexpression with associated reduced interferon gamma signature and increased adenosine production which has been associated with a more resistant immunophenotype $(33,34)$. EGFR mutated tumours also have a mutational signature with decreased production of neoantigens and less clonal expansion $(30,35)$. This represents an important clinical finding as high TMB correlates with improved progression free survival (PFS), objective response rate (ORR) and duration of response to immunotherapy (27). Although TMB increases with subsequent therapies, it is more likely that the neoantigen increase and neoepitopes that are generated are polyclonal and therefore less immunogenic.

\section{Single agent immunotherapy}

Patients with EGFR mutations performed poorly in initial phase three studies comparing immunotherapy to docetaxel (36-38). In the CheckMate 057, Keynote 010, OAK and POPLAR studies, cohorts with EGFR mutations were 82, 86,85 and 18 patients respectively. A small subset of the total trial population, but with universally poorer outcomes. This finding was supported in a retrospective review and a metanalysis which yielded poor response rates and lack of overall survival (OS) benefit (HR 1.11; 95\% CI, 0.80-1.53) when compared to chemotherapy $(28,39)$.

In a small initial study, Lisberg et al. assessed the role of pembrolizumab as first line treatment prior to EGFR TKI and reported no objective responses in 10 patients even within a PD-L1 enriched cohort (PD-L1 $\geq 50 \%$ ) of seven patients. The study was discontinued due to futility $(40,41)$.

\section{Combination approaches}

\section{TKI + immunotherapy}

In order to improve response rates to immunotherapy numerous early phase studies have employed combination approaches with TKIs, however several safety concerns have been flagged by this approach and few trials have progressed beyond phase one. Yang and colleagues looked at standard dosing erlotinib or gefitinib in combination with pembrolizumab every two weeks in EGFR-mutant patients enrolled in cohorts E and F of KEYNOTE-021. Enrolment in the gefitinib cohort was however suspended after $71.4 \%$ $(5 / 7)$ of patients developed grade three or four aspartate aminotransferase (AST)/alanine aminotransferase (ALT) elevations deeming the combination not feasible. The erlotinib cohort $(\mathrm{n}=12)$ however was deemed feasible with no grade four toxicities (42). Despite being more tolerable than the gefitinib combination the ORR was $41.7 \%$ which is lower than what historical controls have demonstrated with erlotinib in the first line setting (30). An early phase trial by Creelan et al. also demonstrated significant toxicity of gefitinib in combination with durvalumab (43). A further phase one study evaluating erlotinib with nivolumab in a cohort of 20 patients demonstrated a similar adverse event profile and low ORR of $15 \%$ (44). Further safety signals were raised in the TATTON trial where the combination of osimertinib and durvalumab in a TKI naïve population saw a $38 \%$ rate of interstitial lung disease (45). A planned phase III study by Yang et al. (CAUREL study) with a similar osimertinib and durvalumab arm had recruitment terminated early secondary to these findings limiting comparisons between the two groups (46). In contrast to the aforementioned studies a phase I study evaluating the combination of atezolizumab and erlotinib yielded a response rate of $75 \%(n=20)$ and more manageable safety profile (47).

Overall these early studies (see Table 1) demonstrated that combination TKI plus immunotherapy approaches were 
Table 1 Early phase studies for immunotherapy in EGFR mutated lung cancers

\begin{tabular}{|c|c|c|c|c|}
\hline Author, phase & Intervention & $\begin{array}{l}\mathrm{N}=\text { number of } \\
\text { participants }\end{array}$ & $\begin{array}{c}\text { Response } \\
\text { rates \% }\end{array}$ & Toxicity \\
\hline \multicolumn{5}{|l|}{ EGFR positive } \\
\hline $\begin{array}{l}\text { Gettinger et al. } \\
\text { [2018], phase I }\end{array}$ & $\begin{array}{l}\text { TKI: Erlotinib; ICl: } \\
\text { Nivolumab }\end{array}$ & 20 & 15 & $\begin{array}{l}\text { G3 events - 25\% [5] } \\
\text { - Raised AST [1] } \\
\text { - Raised ALT [1] } \\
\text { - Diarrhoea [2] } \\
\text { - Weight loss [1] }\end{array}$ \\
\hline $\begin{array}{l}\text { Rudin et al. [2018], } \\
\text { Phase lb }\end{array}$ & $\begin{array}{l}\text { TKI: Erlotinib; ICI: } \\
\text { Atezolizumab }\end{array}$ & 28 & 75 & $\begin{array}{l}\text { G3 } \mathrm{AE} \text { in } 43 \% \\
\text { - ALT rise } 2 \\
\text { - Pyrexia } 2 \\
\text { - Rash } 2 \\
\text { - Diarrhoea } 2\end{array}$ \\
\hline
\end{tabular}

TKI, tyrosine kinase inhibitor, ICI, immune checkpoint inhibitor; G3, grade 3; G4, grade 4; AE, adverse event.

not associated with any consistent benefit but potentially increased toxicities in EGFR-mutant NSCLC.

\section{Chemotherapy + immunotherapy}

Although several combination chemotherapy plus immunotherapy studies excluded EGFR-mutant tumours, the IMPOWER150 study was an exception after the protocol was amended to include patients who had failed prior TKIs. The trial enrolled 1,202 patients overall with equal randomisation to one of three arms: atezolizumab/ carboplatin/paclitaxel (arm A), atezolizumab/carboplatin/ paclitaxel/bevacizumab (arm B) and carboplatin/paclitaxel/ bevacizumab (arm C).

The exploratory analysis included 124 patients with EGFR mutation positive tumours [approximately $10 \%$ of in the intention-to-treat (ITT) population] of which 91 had sensitising mutations and 78 had progressed on prior TKIs. Forty-five, 34 and 45 patients were allocated to arms A, B and C respectively. There was a trend towards median OS improvement in arm B (NE) versus arm C (18.7 months) with a HR of 0.61 (95\% CI, 0.29-1.28) as well as in median PFS (10.2 vs. 6.9 months; HR 0.61 ; $95 \%$ CI, $0.36-1.03)$. The ORR was $36 \%, 71 \%$ and $42 \%$ in groups A,
$\mathrm{B}$ and $\mathrm{C}$ respectively. No trend for OS was noted between arm $\mathrm{A}$ and $\mathrm{C}$ suggesting bevacizumab was an important component of these arms $(48,49)$. Safety results were similar between the EGFR-mutant and ITT populations. While these results are promising, the small numbers were underpowered to establish a benefit or determine a difference between the treatment regimens for this cohort. A further factor limiting clinical applicability is that only 10 patients were previously treated with osimertinib, an established standard of care in either the first or second line T790M setting.

The addition of bevacizumab is thought to have an immunomodulatory effect in this setting and in melanoma has been found to increase circulating CD8+ $\mathrm{T}$ cells and decrease interleukin- 6 and in combination with chemotherapy may assist in changing the tumour microenvironment from "cold" to "hot" $(30,50)$. There is evidence for intertwining roles of the VEGF and EGFR pathways with downstream EGFR activity having been reported to increase VEGF expression and inhibition of VEGF pathways being associated with reduced EGFR activity (51). A strength of IMPOWER150 was that it looked at the addition of VEGF inhibition and thus this 
relationship. The absence of a survival benefit in the ACP group supports this therapeutic pathway but it needs to be further explored.

The approach of combining chemotherapy with immunotherapy will also be looked at in an additional up and coming phase three trial, Keynote-789, where pembrolizumab is added to platinum doublet chemotherapy post failure on first line TKI and in the ABC-Lung phase two study investigating the combination of atezolizumab and bevacizumab with chemotherapy.

\section{Immunotherapy combinations}

While PDL-1 and CTLA4 represent two independent pathways that can be targeted by immune checkpoint inhibition and targeting them in combination has been shown to have synergistic responses, the major lung studies investigating this, Checkmate-227 and MYSTIC, excluded EGFR mutant patients (52). The Illuminate and Checkmate-772 studies will investigate durvalumab/ tremelimumab and nivolumab/ipilimumab respectively in this context.

\section{Summary}

For EGFR-mutant tumours it is clear that single agent immunotherapy and TKI combinations with immunotherapy do not offer any benefit for the majority of patients. Combination strategies are the most promising approach, however, the only prospective data comes from an underpowered subset analysis suggesting chemotherapy plus bevacizumab and atezolizumab was superior to chemotherapy and bevacizumab alone. Results from further clinical trials specifically powered to address this question are eagerly awaited.

\section{Anaplastic lymphoma kinase (ALK) rearrangements}

\section{Current practice and the limitations of TKIs}

$A L K$ gene fusions are present in 3-7\% of lung adenocarcinomas and serve as an important oncogene (53). Crizotinib was quickly identified as an effective agent in targeting $A L K$ rearranged tumours in the PROFILE 1007 study (10) with the registrational PROFILE 1014 study demonstrating a superior response rate $(74 \%$ vs. $45 \%)$ as well as PFS (10.9 vs. 7.0 months) for crizotinib over chemotherapy (54). As with EGFR-mutant tumours resistance inevitably develops and further approved therapies such as ceritinib and alectinib have been developed with success in extending the median time to progression with a recent update in the ALEX study confirming median PFS times of 34.8 months with alectinib compared with 10.9 months with crizotinib (11).

Similarly to EGFR mutant NSCLC, the role of immunotherapy in $A L K$ rearranged lung cancers initially suggested potential benefit.

\section{Combination approaches}

\section{TKI + immunotherapy}

An early phase study by Spigel et al. looking at the combination of nivolumab plus crizotinib as a first line treatment in ALK rearranged patients was notable for $38 \%$ $(n=5)$ developing severe hepatotoxicity which resulted in closure of the cohort (55). After the interim safety review two patients developed severe hepatoxicity and died. Of those who were evaluable five patients (38\%) had partial response, two (15\%) had stable disease and three $(23 \%)$ progressed (56). The phase Ib JAVELIN 101 trial had an ALK positive cohort treated with avelumab and lorlatinib in combination after progression on initial ALK TKI (57). In contrast to the study performed by Spigel $e t a l$. there were no dose limiting toxicities with an ORR 46.4\% $(55,57)$. The combination of alectinib and atezolizumab has also been evaluated (58). ORR was $81 \%$ and duration of response 21.7 months which is still below that of the reported duration of response to single agent alectinib in ALEX (11). Felip and colleagues have also looked at the combination of nivolumab and ceritinib in 36 ALK rearranged patients where $25 \%$ experienced at least a grade three elevation in ALT and 22\% in GGT (59). A safety signal resulted in a protocol amendment resulting in sequential therapy. Recently, the ATLANTIC trial also reported on a cohort of 15 patients (out of a total 111) with ALK rearrangements who received third line or later durvalumab; none of whom had an objective response (60). Table 2 summarises the main results from these early phase studies.

\section{Chemotherapy + immunotherapy}

As with $E G F R$-mutant lung cancer there are numerous theories as to why $A L K$ rearranged tumours have had disappointing responses to immune checkpoint blockade but the underlying reasons remain unclear. The lack of a smoking phenotype and low TMB have been proposed. It is for these reasons in addition to a lack of safety signals that 
Table 2 Early phase studies for immunotherapy in ALK rearranged lung cancers

\begin{tabular}{|c|c|c|c|c|}
\hline Author, phase & Intervention & $\begin{array}{l}\mathrm{N}=\text { number of } \\
\text { participants }\end{array}$ & Response rates \% & Toxicity \\
\hline \multicolumn{5}{|l|}{ ALK positive } \\
\hline Kim et al. [2018] & $\begin{array}{l}\text { TKI: Alectinib; ICl: } \\
\text { Atezolizumab }\end{array}$ & 21 & 81 & G3 62\%; Rash/ALT rise/Pneumonitis \\
\hline $\begin{array}{l}\text { Felip et al. [2020], } \\
\text { Phase Ib }\end{array}$ & $\begin{array}{l}\text { TKI: Ceritinib, ICI: } \\
\text { Nivolumab }\end{array}$ & 36 & $\begin{array}{l}\text { First line } 450 \text { mg: } 83 \text {; } \\
300 \text { mg: } 60 \text {; Second line } \\
450 \text { mg: } 50 ; 300 \text { mg: } 25\end{array}$ & ALT rise $25 \%$; GGT rise $22 \%$; Amylase $14 \%$ \\
\hline
\end{tabular}

TKI, tyrosine kinase inhibitor; ICI, immune checkpoint inhibitor; G3, grade 3; G4, grade 4.

have made it difficult for combination immunotherapy and TKI approaches to proceed beyond the early phase setting.

The IMPOWER150 study, post protocol amendment, also allowed ALK positive patients to be recruited. The addition of atezolizumab to the combination of bevacizumab, carboplatin and paclitaxel improved PFS and OS in the ITT population. However, these cohorts only included 13 ALK positive patients in the quadruplet cohort and 21 in the control. The data for EGFR and ALK were combined as discussed above, making it difficult to interpret the specific benefit in the ALK population although it remains an FDA approved treatment option for both populations.

\section{Summary}

For $A L K$ rearranged NSCLC little benefit has been seen with immunotherapy and TKI combinations. Chemotherapy and immunotherapy combinations represent a promising approach however application of clinical trials is often limited by the exclusion of these patients or small cohorts, as was the case for IMPOWER150.

\section{KRAS mutations}

$K R A S$ is the most common proto-oncogene associated with NSCLC in western populations. It has a heterogeneous distribution according to ethnic origin; having been described in $26.1 \%$ of lung adenocarcinomas and $6.4 \%$ of squamous cell carcinomas in Western countries and in
$11.2 \%$ and $1.8 \%$ of lung cancer cases in Asia respectively $(2,61,62)$. Traditionally it has been associated with smoking (63) and considered a poor prognostic biomarker (64); however, current data indicates a limited effect on OS in patients with early-stage NSCLC (65). The most frequent oncogenic KRAS mutations in NSCLC are missense substitutions occurring at codons 12 and 13: G12C (40\%), G12V (20\%), and G12D (20\%) (66).

\section{KRAS-mutant NSCLC: not a single disease}

Growing preclinical and clinical evidence suggests that $K R A S$-mutant NSCLC might not be a unique entity, and translational studies have unravelled some of the main determinants of this biological diversity. Perhaps this heterogeneity is related to the unfavourable outcomes seen when targeting this pathway in the past. Only recently, a type-specific G12C KRAS inhibitor has shown for the first time some clinically meaningful efficacy for patients with KRAS-mutant NSCLC (67).

Unlike other oncogene-driven lung cancers, KRASmutant lung tumours frequently appear with other major genetic co-mutations. Skoulidis et al. identified three robust expression based clusters in such malignancies: 'KL' subgroup, with STK11 and KEAP co-mutations, 'KP' subgroup, with TP53 co-mutations, and ' $\mathrm{KC}$ ' subgroup, with CDKN2A/B inactivation plus low thyroid transcription factor-1 (TTF-1) (68). 'KC' tumours showed enrichment of gene expression signatures reflecting both upper and lower gastrointestinal neoplastic processes and wild-type TP53 
transcriptional activity. 'KP' subgroup was characterised by active inflammation with high levels of PD-L1 expression. 'KL' tumours were mostly immune inert tumours, with moderate T-cell inflammation and weak PD-L1 expression. Furthermore, co-occurring STK11 and KEAP1 mutations with $K R A S$ appeared to be associated with worse OS $(68,69)$. Hence, the presence of major co-occurring genetic events might also predict distinct therapeutic vulnerabilities in addition to their prognostic significance.

\section{Rationale for immunotherapy}

There is preclinical rationale supporting immunotherapy use in KRAS-mutant NSCLC. PD-L1 expression appears to be intrinsically rather than adaptively elevated, via activation of downstream KRAS signalling pathways (70,71). Besides its relation with KRAS-mutant NSCLC, tobacco smoke induces PD-L1 expression and renders the exposed cells able to evade the immune system and promote lung carcinogenesis (72), and smoking-associated lung cancers have a high mutational burden and abundant T-cell infiltration (73). Huynh et al. described up to $50 \%$ of KRAS-mutant NSCLC with some PD-L1 expression which was correlated both with smoking history and high T-cell infiltration (74). Nevertheless, it is important to consider the previously mentioned heterogeneity. KRAS-mutant NSCLCs display different immune profiles and could consequently have varying levels of sensitivity to immunotherapy (68). In the case of KRAS-TP53 co-mutated tumours, a higher mutational burden and genomic instability could explain, at least partially, the T-cell-inflamed microenvironment and the adaptive immune-resistant phenotype $(68,75)$. KRASSTK11 co-mutant subgroups have been associated with high hypoxia-inducible factor 1 (HIF1 $\alpha$ ), which leads to impaired T-cell function (76). In a retrospective pooled analysis, including three independent subgroups $(\mathrm{n}=174)$ and a subset of patients with KRAS-mutant lung adenocarcinomas $(\mathrm{n}=44)$ all treated with ICIs, patients with KRAS-STK11 co-mutations demonstrated a significantly lower ORR, PFS and OS than patients with KRAS-TP53 tumours (77); furthermore, $S T K 11$ was associated with resistance to PD-1 blockade in PD-L1 positive NSCLC, regardless of KRAS mutational status (77). This data suggests that despite ICIs being one of the most promising biological therapies for $K R A S$-mutant NSCLC, they do not benefit all patients equally. A potential means of predicting immunoresistance in these patients may involve consideration of the heterogeneity of KRAS-mutant tumours, particularly the presence of significant co-mutations.

\section{Clinical evidence}

The current clinical evidence to support treatment in NSCLC based solely on a KRAS mutation is confounding. In an extensive retrospective registry in NSCLC with common driver mutations treated with ICIs, almost half of patients had KRAS mutations $(\mathrm{N}=271$ of 551$)$, with an overall cohort best response of $19 \%$, and median PFS of 2.8 months (mainly driven by the large KRAS-subgroup) (78) (Table 3). When using prospective data, in an unplanned subgroup analysis of trials comparing ICIs such as nivolumab or atezolizumab with chemotherapy in second-line treatment for NSCLC, it was suggested that KRAS mutations were more sensitive to ICIs compared with wild-type and that ICIs as second or third-line therapy in KRAS-mutant NSCLC improved OS $(36,38,79)$. For instance, in the CheckMate-057 trial, KRAS-mutant subsets $(\mathrm{n}=62)$ were among the molecular subgroups to achieve the most significant OS benefits with nivolumab (HR 0.52; 95\% CI, 0.29-0.95) (36). A meta-analysis (80) including these trials investigated the predictive role of KRAS-mutations in 519 patients with previously treated NSCLC, resulting in a greater benefit in the KRASmutant subgroup (HR, 0.65; 95\% CI, 0.44-0.97; $\mathrm{P}=0.03$ ). Individually, these studies were not powered to address this question, and KRAS-mutation status was known only in a small fraction of cases, undermining the clinical validity of this finding. Furthermore, using prospective 'real-world' observational patient data, two studies have concluded that KRAS mutation status did not confer significant differences in ORR, PFS and OS $(81,82)$. A summary of these studies can be found in Table 3. In Figure 2 we also provide an example of a patient with metastatic NSCLC who had a KRAS G12C mutation and TP53 co-mutation with complete response post three cycles of immunotherapy.

\section{Summary}

Immunotherapy is one of the most promising new therapies in KRAS-mutant NSCLC, but recent data suggests variable efficacy of immunotherapy according to the presence of other co-mutations. The targetability of $K R A S$ G12C mutation also adds further complexity when considering which patients are most likely to benefit from 
Table 3 Retrospective studies of ICIs in KRAS and BRAF mutant NSCLC

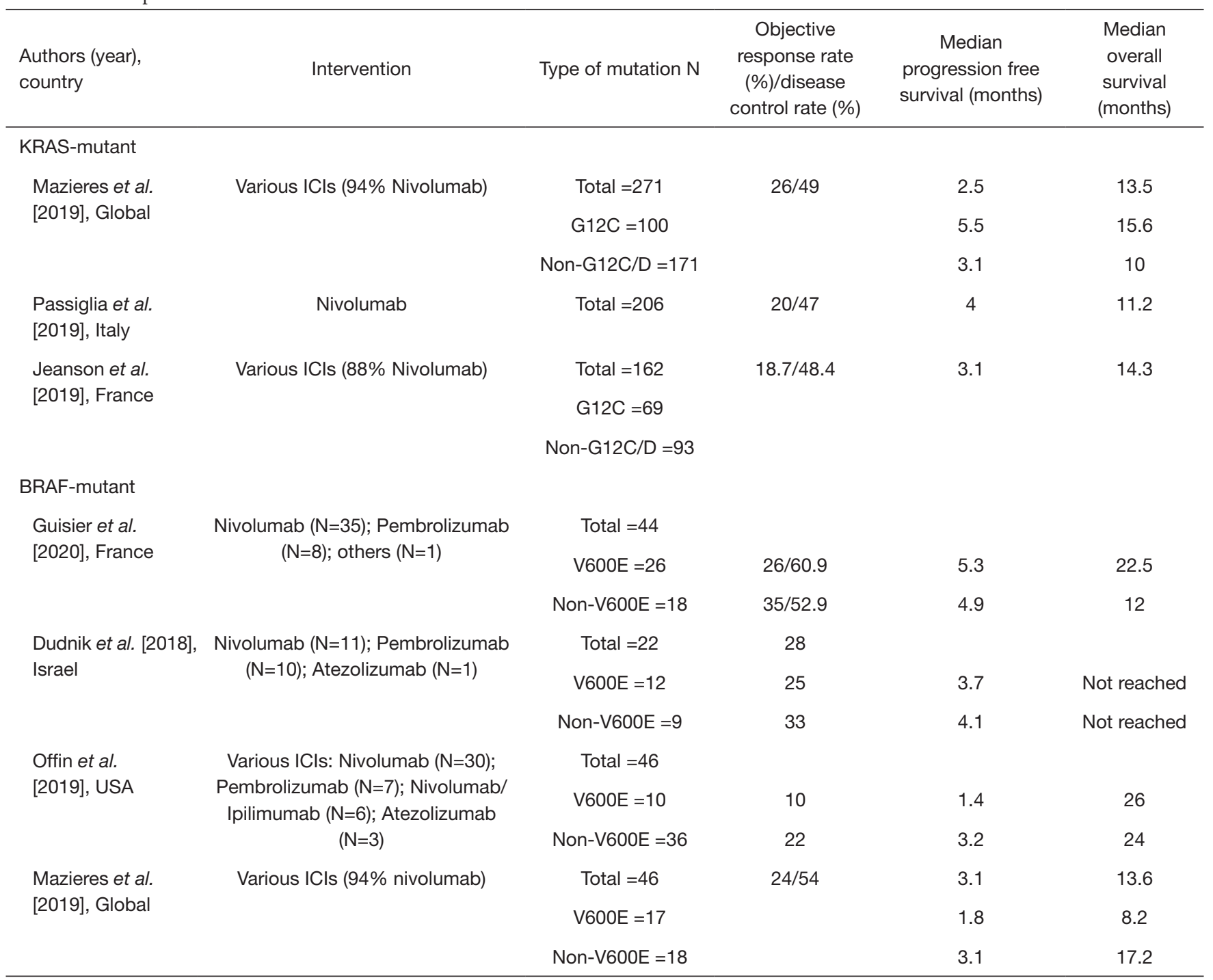

$\mathrm{ICl}$, immune checkpoint inhibitor.

targeted therapies compared to ICIs. Given the biological heterogeneity of KRAS-mutant NSCLC, treatment will likely need to be individualized and may require combinations of treatment, many of which are currently under investigation.

\section{BRAF mutations}

BRAF mutations result in persistent activation of downstream cell signalling through the MAPK pathway and lead to unchecked cell growth and proliferation (83). The most common variant responsible for this process is a BRAF point mutation V600E and has been described with variable frequency in melanoma, colorectal cancer, papillary thyroid cancer, among others malignancies (84). BRAF mutations are uncommon in NSCLC, occurring in $1-5 \%$ of cases (85-88). Large clinicopathologic studies show some variability relating to the specific clinical characteristics associated with BRAF mutations (85-88). Overall, studies suggest slightly increased frequency in females. Smoking and its link with BRAF status has varied among studies, however in Caucasians with a positive smoking history there is also an association with sarcomatoid histology and both non-V600E and V600E BRAF mutations (85-87). Across all studies, the main consistent finding was poor ORR, PFS and disease-free survival (DFS) in patients 
A

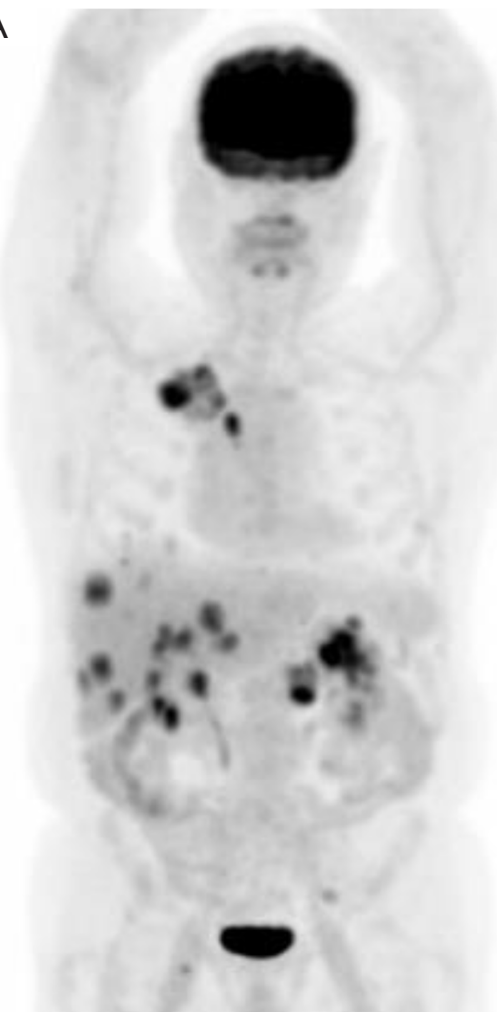

B

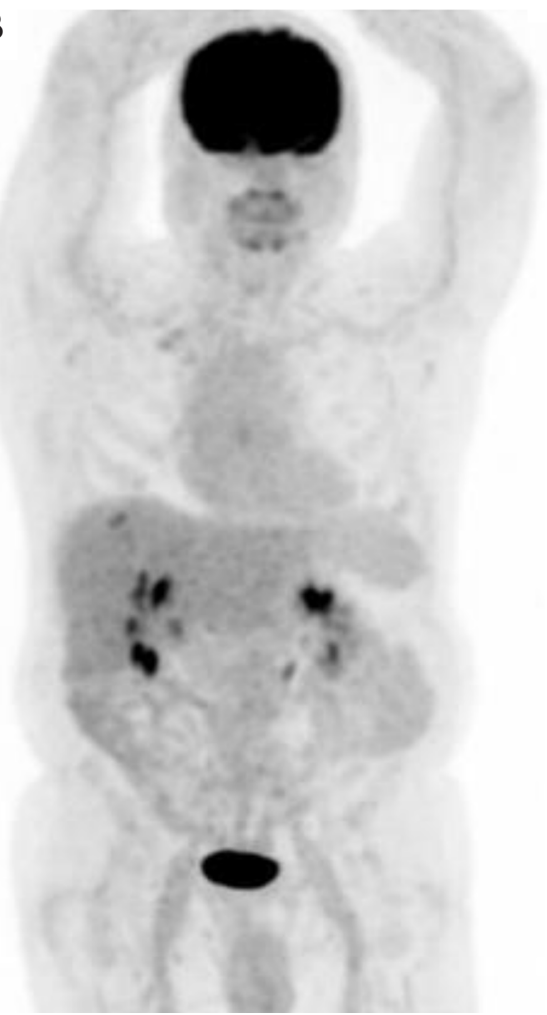

C

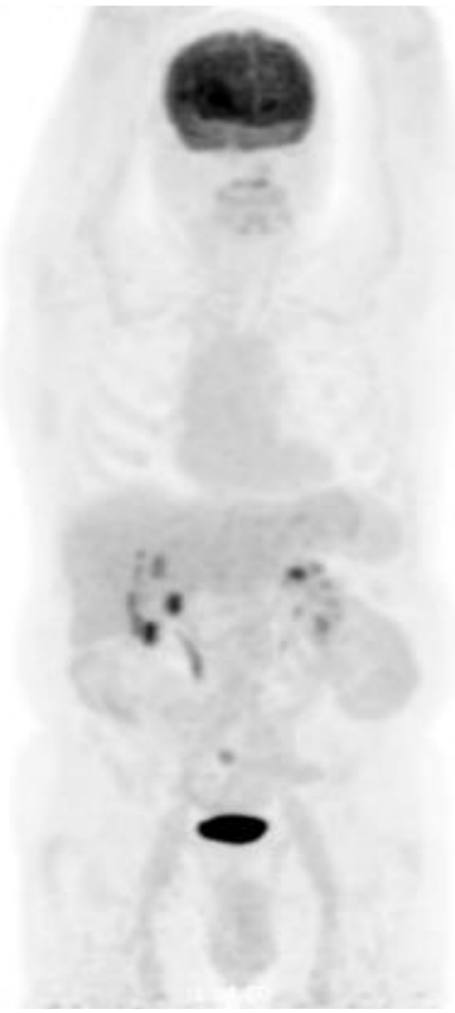

Figure 2 A 72-year-old old male, heavy smoker (40 pack-years). Metastatic non-small cell lung cancer: PD-L1 90\%, KRAS G12C mutation and TP53 co-mutation. Commenced on pembrolizumab $200 \mathrm{mg}$ 3-weekly. Positron emission tomography (PET): (A) baseline PET prior staring therapy shows primary tumour on the upper right lobe and extensive hepatic, retroperitoneal and right kidney metastases. (B) PET CT after three cycles of pembrolizumab showing almost complete metabolic response. (C) PET CT after 36 months of starting pembrolizumab which shows an ongoing complete response.

with a BRAF V600E mutation treated with chemotherapy when compared with non-V600E mutants (86-88).

\section{Current practice and the limitations of TKIs}

Like melanoma, BRAF V600E NSCLC has emerged as an important target for drug therapy with BRAF inhibitors (89). Since resistance invariably develops with BRAF inhibition alone, via the development of bypass pathways such as redirection of cell signalling via MEK $1 / 2$ kinases, adding a MEK inhibitor improved clinical outcomes in both melanoma and NSCLC (89).

The data supporting the use of a BRAF inhibitor with or without MEK inhibition in BRAF positive NSCLC has been adopted from a small number of positive phase two studies. In a phase two, multi-cohort, non-randomized, open-label study, 78 previously treated BRAF V600 mutation-positive NSCLC patients received dabrafenib; responses were observed in $33 \%$, the median PFS was 5.5 months (95\% CI, 3.4-7.3) and median OS 12.7 months (95\% CI, 7.3-16.9) (90). A second cohort of the same study evaluated the combination of dabrafenib/trametinib with an ORR of $63 \%$ and median PFS of 9.7 months when compared to single-agent dabrafenib (91). The median OS was also 12.7 months in the dabrafenib monotherapy cohort versus 18.2 months in the dabrafenib/trametinib cohort (91). Vemurafenib was tested in another multi-tumour phase two study where responses were seen in $42 \%$, the median PFS was 7.3 months (95\% CI, 3.5-10.8) and median OS had not been reached (92).

\section{Immunotherapy in BRAF-mutant NSCLC}

Evidence to support immunotherapy in $B R A F$ mutated NSCLC comes only from retrospective evidence. A retrospective review included $39 B R A F$-mutated NSCLC patients, stratified by PD-L1 status, TMB and 
microsatellite instability (MSI), but not all patients were tested for each of these markers. Twenty-two received an ICI: $57 \%$ of the V600E group and $55 \%$ of the nonV600E group. ORR was $25 \%$ and $33 \%$ and median PFS 3.7 and 4.1 months respectively. PD-L1 status and $B R A F$ mutation type did not alter the outcome, and the study suggested that $B R A F$-mutated NSCLC was more likely to have high expression of PD-L1; a point to interpret with caution given the low number of cases $(27,93)$. Another retrospective study addressing the efficacy of ICIs in Japanese patients with oncogenic driver mutations, reported only five cases with a BRAF mutated NSCLC, with no responses observed (94). The most substantial report comes from a recent publication by Guisier $e t$ al. of a retrospective review from several centres in France. They detected 44 patients with $B R A F$ mutations receiving ICIs (V600: 26, non-V600: 18). The ORR, median PFS, OS, and 12-month OS for BRAF V600E and BRAF non-V600E were $26 \%$ and $35 \%, 5.3$ and $4.9,22$ and 12 months, $53 \%$ and $44 \%$ respectively (95). Less than half of the patients had a PD-L1 status assessment and thus no conclusions were drawn. A brief summary of these reports is available in Table 3.

\section{Summary}

Overall based on the current literature it seems that, in contrast to other oncogene addicted NSCLCs, ICIs seem to have some activity in those with a $B R A F$ mutation. This activity is similar to that observed in patients with pretreated unselected NSCLC in randomised controlled trials or observational studies and seems to be irrespective of PD-L1 status or BRAF mutation type. However, BRAF mutations can occur in heavy smokers and also in nonsmokers and it is likely that the best responses are driven by the smoking phenotype although current data are limited.

\section{Other oncogene addicted tumours}

\section{c-ROS oncogene 1 (ROS1) rearrangements/Human epidermal growth factor receptor 2 (HER2)/RET/MET}

Since discovery in 2007 ROS1 rearrangements have become another important target in NSCLC and are identified in $1-2 \%$ of patients (96). The recently published Immunotarget trial (78) was a retrospective study evaluating single agent immune checkpoint inhibition in advanced NSCLC across numerous oncogenic alterations. They reviewed 551 patients and evaluated their molecular alterations: $K R A S(\mathrm{n}=271), E G F R(\mathrm{n}=125), B R A F(\mathrm{n}=43)$, $\operatorname{MET}(\mathrm{n}=36), \operatorname{HER} 2(\mathrm{n}=29), A L K(\mathrm{n}=23), \operatorname{RET}(\mathrm{n}=16)$, ROS1 $(\mathrm{n}=7)$ and multiple drivers $(\mathrm{n}=1)$. They then evaluated clinicopathologic characteristics and outcomes for immune checkpoint blockade. In the overall cohort the best response with immunotherapy was $19 \%$ with a median PFS of 2.8 months. Most of this benefit appeared to be driven by the KRAS cohort. For the rarer oncogenic addicted tumours, which expectedly had small numbers, this study represents the limited data evaluating ICI use in this setting. For HER2-mutant tumours a median PFS of 2.5 months (95\% CI, 1.8-3.5) was noted and 2.1 months (95\% CI, 1.34.7) for RET rearranged NSCLC. Only seven patients had ROS1 rearrangements and a median PFS was not reported.

Findings for tumours with MET alterations [median PFS 3.4 months (95\% CI, 1.7-6.2)] were in keeping with a study by Sabari et al. (97) which demonstrated only a modest benefit to immune checkpoint inhibition with an ORR of $17 \%$ and median PFS 1.9 months (95\% CI, 1.7-2.7) in a cohort of 24 patients who had received prior chemotherapy. Sabari and colleagues also evaluated PD-L1 expression in their study but found no correlation to treatment response. TMB was also found to be low in these patients. These response rates sit well below the reported $32 \%$ ORR and 7.3 months median PFS seen with crizotinib in the PROFILE 1001 study (98) and newer TKIs such as capmatinib, tepotinib and savolitinib are also reporting high responses for this population (99-101).

Overall response rates appear to be very modest to immunotherapy in these rarer oncogene addicted tumours with most benefit being derived by the KRAS cohort. The approach for the rare patients with multiple alterations such as additional TP53 or PIK3Ca mutations is uncertain and requires further research (102). Pursuing other systemic therapies or TKIs for these tumours is recommended and ongoing trials are required to review whether combination approaches (such as the addition of immunotherapy to chemotherapy) may improve outcomes for these patients.

\section{Conclusions}

Oncogene addicted tumours while heterogeneous, collectively account for over $40 \%$ of non-squamous NSCLCs. It is important to note that most single agent and combination immunotherapy trials that have been reported to date excluded specific oncogene addicted 
NSCLCs such as ALK and EGFR; however, it is likely that other oncogene driven tumours were not tested for but included in the non-smoker subsets. Trials that focused on these tumours have largely been negative. However, of key importance is that the use of chemotherapy in combination with immunotherapy may provide benefits to this approach not seen with single agent ICIs.

Whilst it is clear for most non-smoking related oncogene addicted tumours that immunotherapy should only be used in combination, the actual data underpinning this approach remains limited. It is more likely that these tumours develop immunoevasion abilities through mechanisms which PD-1/CTLA4 blockade does not abrogate. It is clear that a better understanding of these pathways is needed in order to develop robust markers and targets for therapies so that the immunotherapy benefits seen in some smoking associated NSCLC can also be realised in this patient group.

Best evidence to date supports the use of TKI therapy prior to immunotherapy or chemotherapy in oncogene addicted tumours and clinical trials should always be considered for this population of patients. Combination approaches with chemotherapy and immunotherapy in EGFR positive or ALK rearranged tumours post TKI exhaustion appears to be most promising as suggested by the IMpower150 data. The role of biopsy in progressing disease has largely been limited to the identification of T790M in EGFR mutant disease as a method of accessing osimertinib, however, in the era of precision medicine with the ongoing development and evolution of targeted therapies and as we develop a greater understanding of the mechanisms of resistance to TKI therapy this is may very well change in the future.

\section{Acknowledgments}

Funding: None.

\section{Footnote}

Provenance and Peer Review: This article was commissioned by the Guest Editor (Daniel Steinfort) for the series "Lung cancer and the immune system" published in Translational Lung Cancer Research. The article has undergone external peer review.

Peer Review File: Available at http://dx.doi.org/10.21037/ tlcr-20-772
Conflicts of Interest: All authors have completed the ICMJE uniform disclosure form (available at http://dx.doi. org/10.21037/tlcr-20-772). The series "Lung cancer and the immune system" was commissioned by the editorial office without any funding or sponsorship. JL reports grants and personal fees from MSD (Merck) Roche, Boheringer Ingelheim, Pfizer, Astra Zeneca, BMS and personal fees from Sanofi, Tecnofarma, Elly-Lilly. BS reports Advisory Boards/Honoraria AstraZeneca, Roche/Genentech, Pfizer, Novartis, Merck Shape Dohme, Bristol Myers Squibb, Specialized Therapeutics, Loxo Oncology, Amgen. TJ reports Honoraria: AZ, BMS, Roche, Pfizer, Novartis, Specialised Therapeutics, Amgen, MSD, Merck, Takeda. The authors have no other conflicts of interest to declare.

Etbical Statement: The authors are accountable for all aspects of the work in ensuring that questions related to the accuracy or integrity of any part of the work are appropriately investigated and resolved.

Open Access Statement: This is an Open Access article distributed in accordance with the Creative Commons Attribution-NonCommercial-NoDerivs 4.0 International License (CC BY-NC-ND 4.0), which permits the noncommercial replication and distribution of the article with the strict proviso that no changes or edits are made and the original work is properly cited (including links to both the formal publication through the relevant DOI and the license). See: https://creativecommons.org/licenses/by-nc$\mathrm{nd} / 4.0 /$.

\section{References}

1. Siegel RL, Miller KD, Jemal A. Cancer statistics, 2020. CA Cancer J Clin 2020;70:7-30.

2. Skoulidis F, Heymach JV. Co-occurring genomic alterations in non-small-cell lung cancer biology and therapy. Nat Rev Cancer 2019;19:495-509.

3. Jordan EJ, Kim HR, Arcila ME, et al. Prospective Comprehensive Molecular Characterization of Lung Adenocarcinomas for Efficient Patient Matching to Approved and Emerging Therapies. Cancer Discov 2017;7:596-609.

4. Frampton GM, Ali SM, Rosenzweig M, et al. Activation of MET via diverse exon 14 splicing alterations occurs in multiple tumor types and confers clinical sensitivity to MET inhibitors. Cancer Discov 2015;5:850-9.

5. Lee CK, Davies L, Wu YL, et al. Gefitinib or Erlotinib vs 
Chemotherapy for EGFR Mutation-Positive Lung Cancer: Individual Patient Data Meta-Analysis of Overall Survival. J Natl Cancer Inst 2017;109:10.1093/jnci/djw279..

6. Mok TS, Wu YL, Ahn MJ, et al. Osimertinib or PlatinumPemetrexed in EGFR T790M-Positive Lung Cancer. N Engl J Med 2017;376:629-40.

7. Sequist LV, Yang JC, Yamamoto N, et al. Phase III study of afatinib or cisplatin plus pemetrexed in pa-tients with metastatic lung adenocarcinoma with EGFR mutations. J Clin Oncol 2013;31:3327-34.

8. Ramalingam SS, Vansteenkiste J, Planchard D, et al. Overall Survival with Osimertinib in Untreated, EGFR-Mutated Advanced NSCLC. N Engl J Med 2020;382:41-50.

9. Planchard D, Smit EF, Groen HJM, et al. Dabrafenib plus trametinib in patients with previously un-treated BRAFV600E-mutant metastatic non-small-cell lung cancer: an open-label, phase 2 trial. Lancet Oncol 2017;18:1307-16.

10. Shaw AT, Kim DW, Nakagawa K, et al. Crizotinib versus chemotherapy in advanced ALK-positive lung cancer. $\mathrm{N}$ Engl J Med 2013;368:2385-94.

11. Camidge DR, Dziadziuszko R, Peters S, et al. Updated Efficacy and Safety Data and Impact of the EML4-ALK Fusion Variant on the Efficacy of Alectinib in Untreated ALK-Positive Advanced Non-Small Cell Lung Cancer in the Global Phase III ALEX Study. J Thorac Oncol 2019;14:1233-43.

12. Solomon BJ, Besse B, Bauer TM, et al. Lorlatinib in patients with ALK-positive non-small-cell lung cancer: results from a global phase 2 study. Lancet Oncol 2018;19:1654-67.

13. Shaw AT, Ou SH, Bang YJ, et al. Crizotinib in ROS1rearranged non-small-cell lung cancer. N Engl J Med 2014;371:1963-71.

14. Ackermann CJ, Stock G, Tay R, et al. Targeted Therapy For RET-Rearranged Non-Small Cell Lung Cancer: Clinical Development And Future Directions. Onco Targets Ther 2019;12:7857-64.

15. Tan DS, Mok TS, Rebbeck TR. Cancer Genomics: Diversity and Disparity Across Ethnicity and Geog-raphy. J Clin Oncol 2016;34:91-101.

16. Janne PA, Yang JC, Kim DW, et al. AZD9291 in EGFR inhibitor-resistant non-small-cell lung cancer. N Engl J Med 2015;372:1689-99.

17. Ettinger DS, Wood DE, Aisner DL, et al. Non-Small Cell Lung Cancer, Version 5.2017, NCCN Clini-cal Practice Guidelines in Oncology. J Natl Compr Canc Netw 2017;15:504-35.
18. Novello S, Barlesi F, Califano R, et al. Metastatic nonsmall-cell lung cancer: ESMO Clinical Practice Guidelines for diagnosis, treatment and follow-up. Ann Oncol 2016;27:v1-v27.

19. Rosell R, Carcereny E, Gervais R, et al. Erlotinib versus standard chemotherapy as first-line treatment for European patients with advanced EGFR mutation-positive non-small-cell lung cancer (EURTAC): a multicentre, open-label, randomised phase 3 trial. Lancet Oncol 2012;13:239-46.

20. Zhou C, Wu YL, Chen G, et al. Erlotinib versus chemotherapy as first-line treatment for patients with advanced EGFR mutation-positive non-small-cell lung cancer (OPTIMAL, CTONG-0802): a multicen-tre, open-label, randomised, phase 3 study. Lancet Oncol 2011;12:735-42.

21. Mitsudomi T, Morita S, Yatabe Y, et al. Gefitinib versus cisplatin plus docetaxel in patients with non-small-cell lung cancer harbouring mutations of the epidermal growth factor receptor (WJTOG3405): an open label, randomised phase 3 trial. Lancet Oncol 2010;11:121-8.

22. Inoue A, Kobayashi K, Maemondo M, et al. Updated overall survival results from a randomized phase III trial comparing gefitinib with carboplatin-paclitaxel for chemonaive non-small cell lung cancer with sen-sitive EGFR gene mutations (NEJ002). Ann Oncol 2013;24:54-9.

23. Wu YL, Zhou C, Liam CK, et al. First-line erlotinib versus gemcitabine/cisplatin in patients with ad-vanced EGFR mutation-positive non-small-cell lung cancer: analyses from the phase III, randomized, open-label, ENSURE study. Ann Oncol 2015;26:1883-9.

24. Akbay EA, Koyama S, Carretero J, et al. Activation of the PD-1 pathway contributes to immune escape in EGFRdriven lung tumors. Cancer Discov 2013;3:1355-63.

25. Chen N, Fang W, Zhan J, et al. Upregulation of PD-L1 by EGFR Activation Mediates the Immune Es-cape in EGFR-Driven NSCLC: Implication for Optional Immune Targeted Therapy for NSCLC Pa-tients with EGFR Mutation. J Thorac Oncol 2015;10:910-23.

26. Azuma K, Ota K, Kawahara A, et al. Association of PDL1 overexpression with activating EGFR muta-tions in surgically resected non small-cell lung cancer. Ann Oncol 2014;25:1935-40.

27. Rizvi NA, Hellmann MD, Snyder A, et al. Cancer immunology. Mutational landscape determines sensi-tivity to PD-1 blockade in non-small cell lung cancer. Science 2015;348:124-8.

28. Gainor JF, Shaw AT, Sequist LV, et al. EGFR Mutations 
and ALK Rearrangements Are Associated with Low Response Rates to PD-1 Pathway Blockade in Non-Small Cell Lung Cancer: A Retrospective Anal-ysis. Clin Cancer Res 2016;22:4585-93.

29. Offin M, Rizvi H, Tenet M, et al. Tumor Mutation Burden and Efficacy of EGFR-Tyrosine Kinase In-hibitors in Patients with EGFR-Mutant Lung Cancers. Clin Cancer Res 2019;25:1063-9.

30. Bruno D, Dowlati A. Immunotherapy in EGFR mutant non-small cell lung cancer: when, who and how? Transl Lung Cancer Res 2019;8:710-4.

31. Dong ZY, Wu SP, Liao RQ, et al. Potential biomarker for checkpoint blockade immunotherapy and treatment strategy. Tumour Biol 2016;37:4251-61.

32. Soo RA, Lim SM, Syn NL, et al. Immune checkpoint inhibitors in epidermal growth factor receptor mu-tant non-small cell lung cancer: Current controversies and future directions. Lung Cancer 2018;115:12-20.

33. Tay RY, Ackermann CJ, Califano R. Immunotherapy in tyrosine kinase inhibitor-naive advanced epi-dermal growth factor receptor-mutant non-small cell lung cancerdriving down a precarious road in driv-er-mutated lung cancer. Transl Lung Cancer Res 2018;7:S377-80.

34. Streicher K, Higgs BW, Wu S, et al. Increased CD73 and reduced IFNG signature expression in relation to response rates to anti-PD-1(L1) therapies in EGFR-mutant NSCLC. J Clin Oncol 2017;35:11505.

35. Miyauchi E, Matsuda T, Kiyotani K, et al. Significant differences in $\mathrm{T}$ cell receptor repertoires in lung adenocarcinomas with and without epidermal growth factor receptor mutations. Cancer Sci 2019;110:867-74.

36. Borghaei H, Paz-Ares L, Horn L, et al. Nivolumab versus Docetaxel in Advanced Nonsquamous Non-Small-Cell Lung Cancer. N Engl J Med 2015;373:1627-39.

37. Herbst RS, Baas P, Kim DW, et al. Pembrolizumab versus docetaxel for previously treated, PD-L1-positive, advanced non-small-cell lung cancer (KEYNOTE-010): a randomised controlled trial. Lancet 2016;387:1540-50.

38. Rittmeyer A, Barlesi F, Waterkamp D, et al. Atezolizumab versus docetaxel in patients with previously treated non-small-cell lung cancer (OAK): a phase 3, openlabel, multicentre randomised controlled trial. Lancet 2017;389:255-65.

39. Lee CK, Man J, Lord S, et al. Checkpoint Inhibitors in Metastatic EGFR-Mutated Non-Small Cell Lung Cancer-A Meta-Analysis. J Thorac Oncol 2017;12:403-7.

40. Velez MA, Burns TF. Is the game over for PD-1 inhibitors in EGFR mutant non-small cell lung cancer? Transl Lung
Cancer Res 2019;8:S339-42.

41. Lisberg A, Cummings A, Goldman JW, et al. A Phase II Study of Pembrolizumab in EGFR-Mutant, PD-L1+, Tyrosine Kinase Inhibitor Naive Patients With Advanced NSCLC. J Thorac Oncol 2018;13:1138-45.

42. Yang JC, Gadgeel SM, Sequist LV, et al. Pembrolizumab in Combination With Erlotinib or Gefitinib as First-Line Therapy for Advanced NSCLC With Sensitizing EGFR Mutation. J Thorac Oncol 2019;14:553-9.

43. Creelan BC, Yeh T, Kim SW, et al. Phase I study of gefitinib (G) + durvalumab (D) for locally ad-vanced/ metastatic non-small cell lung cancer (NSCLC) harbouring epidermal growth factor receptor (EGFR) sensitising mutations. Ann Oncol 2019;30:ii31-ii7.

44. Gettinger S, Hellmann MD, Chow LQM, et al. Nivolumab Plus Erlotinib in Patients With EGFR-Mutant Advanced NSCLC. J Thorac Oncol 2018;13:1363-72.

45. Ahn MJ, Yang J, Yu H, et al. 136O: Osimertinib combined with durvalumab in EGFR-mutant non-small cell lung cancer: Results from the TATTON phase Ib trial. J Thorac Oncol 2016;11:S115.

46. Yang JCH, Shepherd FA, Kim DW, et al. Osimertinib Plus Durvalumab versus Osimertinib Monother-apy in EGFR T790M-Positive NSCLC following Previous EGFR TKI Therapy: CAURAL Brief Report. J Thorac Oncol 2019;14:933-9.

47. Rudin C, Cervantes A, Dowlati A, et al. MA15.02 LongTerm Safety and Clinical Activity Results from a Phase Ib Study of Erlotinib Plus Atezolizumab in Advanced NSCLC. J Thorac Oncol 2018;13:S407.

48. Socinski MA, Jotte RM, Cappuzzo F, et al. Atezolizumab for First-Line Treatment of Metastatic Nonsquamous NSCLC. N Engl J Med 2018;378:2288-301.

49. Rebuzzi SE, Facchinetti F, Tiseo M. Anti-angiogenesis boosts chemo-immunotherapy in patients with EGFR mutations or baseline liver metastases: insights from IMpower150 study. Transl Cancer Res 2019;8:S612-7.

50. Mansfield AS, Nevala WK, Lieser EA, et al. The immunomodulatory effects of bevacizumab on systemic immunity in patients with metastatic melanoma. Oncoimmunology 2013;2:e24436.

51. Hung MS, Chen IC, Lin PY, et al. Epidermal growth factor receptor mutation enhances expression of vascular endothelial growth factor in lung cancer. Oncol Lett 2016;12:4598-604.

52. Hellmann MD, Ciuleanu TE, Pluzanski A, et al. Nivolumab plus Ipilimumab in Lung Cancer with a High Tumor Mutational Burden. N Engl J Med 2018;378:2093- 
104.

53. Soda M, Choi YL, Enomoto M, et al. Identification of the transforming EML4-ALK fusion gene in non-small-cell lung cancer. Nature 2007;448:561-6.

54. Solomon BJ, Mok T, Kim DW, et al. First-line crizotinib versus chemotherapy in ALK-positive lung cancer. N Engl J Med 2014;371:2167-77.

55. Spigel DR, Reynolds C, Waterhouse D, et al. Phase $1 / 2$ Study of the Safety and Tolerability of Nivolumab Plus Crizotinib for the First-Line Treatment of Anaplastic Lymphoma Kinase Translocation - Positive Advanced Non-Small Cell Lung Cancer (CheckMate 370). J Thorac Oncol 2018;13:682-8.

56. Patel M, Jabbour SK, Malhotra J. ALK inhibitors and checkpoint blockade: a cautionary tale of mixing oil with water? J Thorac Dis 2018;10:S2198-201.

57. Shaw AT, Lee SH, Ramalingam SS, et al. Avelumab (antiPD-L1) in combination with crizotinib or lorla-tinib in patients with previously treated advanced NSCLC: Phase $1 \mathrm{~b}$ results from JAVELIN Lung 101. J Clin Oncol 2018;36:9008.

58. Kim DW, Gadgeel SM, Gettinger SN, et al. Safety and clinical activity results from a phase Ib study of alectinib plus atezolizumab in ALK+ advanced NSCLC (aNSCLC). J Clin Oncol 2018;36:9009.

59. Felip E, Braud FGD, Maur M, et al. Ceritinib plus nivolumab (NIVO) in patients (pts) with anaplastic lymphoma kinase positive (ALK+) advanced non-small cell lung cancer (NSCLC). J Clin Oncol 2017;35:2502.

60. Garassino MC, Cho BC, Kim JH, et al. Durvalumab as third-line or later treatment for advanced non-small-cell lung cancer (ATLANTIC): an open-label, single-arm, phase 2 study. Lancet Oncol 2018;19:521-36.

61. Osta BEE, Behera M, Kim S, et al. Characteristics and outcomes of patients (pts) with metastatic KRAS mutant lung adenocarcinomas: Lung Cancer Mutation Consortium (LCMC) database. J Clin Oncol 2017;35:9021.

62. Dearden S, Stevens J, Wu YL, et al. Mutation incidence and coincidence in non small-cell lung cancer: metaanalyses by ethnicity and histology (mutMap). Ann Oncol 2013;24:2371-6.

63. Dogan S, Shen R, Ang DC, et al. Molecular epidemiology of EGFR and KRAS mutations in 3,026 lung adenocarcinomas: higher susceptibility of women to smoking-related KRAS-mutant cancers. Clin Cancer Res 2012;18:6169-77.

64. Mascaux C, Iannino N, Martin B, et al. The role of RAS oncogene in survival of patients with lung can-cer: a systematic review of the literature with meta-analysis. $\mathrm{Br} \mathrm{J}$ Cancer 2005;92:131-9.

65. Shepherd FA, Domerg C, Hainaut P, et al. Pooled analysis of the prognostic and predictive effects of KRAS mutation status and KRAS mutation subtype in early-stage resected non-small-cell lung cancer in four trials of adjuvant chemotherapy. J Clin Oncol 2013;31:2173-81.

66. Wood K, Hensing T, Malik R, et al. Prognostic and Predictive Value in KRAS in Non-Small-Cell Lung Cancer: A Review. JAMA Oncol 2016;2:805-12.

67. Fakih M, O'Neil B, Price TJ, et al. Phase 1 study evaluating the safety, tolerability, pharmacokinetics (PK), and efficacy of AMG 510, a novel small molecule KRASG12C inhibitor, in advanced solid tumors. J Clin Oncol 2019;37:3003.

68. Skoulidis F, Byers LA, Diao L, et al. Co-occurring genomic alterations define major subsets of KRAS-mutant lung adenocarcinoma with distinct biology, immune profiles, and therapeutic vulnerabili-ties. Cancer Discov 2015;5:860-77.

69. Riely GJ, Jordan E, Kim HR, et al. Association of outcomes and co-occuring genomic alterations in pa-tients with KRAS-mutant non-small cell lung cancer. J Clin Oncol 2016;34:9019.

70. Coelho MA, de Carné Trécesson S, Rana S, et al. Oncogenic RAS Signaling Promotes Tumor Immunoresistance by Stabilizing PD-L1 mRNA. Immunity 2017;47:1083-99.e6.

71. Chen N, Fang W, Lin Z, et al. KRAS mutation-induced upregulation of PD-L1 mediates immune escape in human lung adenocarcinoma. Cancer Immunol Immunother 2017;66:1175-87.

72. Wang GZ, Zhang L, Zhao XC, et al. The Aryl hydrocarbon receptor mediates tobacco-induced PDL1 expression and is associated with response to immunotherapy. Nat Commun 2019;10:1125.

73. Spigel DR, Schrock AB, Fabrizio D, et al. Total mutation burden (TMB) in lung cancer (LC) and rela-tionship with response to PD-1/PD-L1 targeted therapies. J Clin Oncol 2016;34:9017.

74. Huynh TG, Morales-Oyarvide V, Campo MJ, et al. Programmed Cell Death Ligand 1 Expression in Resected Lung Adenocarcinomas: Association with Immune Microenvironment. J Thorac Oncol 2016;11:1869-78.

75. Dong ZY, Zhong WZ, Zhang XC, et al. Potential Predictive Value of TP53 and KRAS Mutation Status for Response to PD-1 Blockade Immunotherapy in Lung Adenocarcinoma. Clin Cancer Res 2017;23:3012-24. 
76. Kerr EM, Martins CP. Metabolic rewiring in mutant Kras lung cancer. FEBS J 2018;285:28-41.

77. Skoulidis F, Goldberg ME, Greenawalt DM, et al. STK11/LKB1 mutations and PD-1 inhibitor re-sistance KRAS-mutant lung adenocarcinoma. Cancer Discov 2018;8:822-35.

78. Mazieres J, Drilon A, Lusque A, et al. Immune checkpoint inhibitors for patients with advanced lung cancer and oncogenic driver alterations: results from the IMMUNOTARGET registry. Ann Oncol 2019;30:1321-8.

79. Fehrenbacher L, Spira A, Ballinger M, et al. Atezolizumab versus docetaxel for patients with previously treated non-small-cell lung cancer (POPLAR): a multicentre, open-label, phase 2 randomised controlled trial. Lancet 2016;387:1837-46.

80. Lee CK, Man J, Lord S, et al. Clinical and Molecular Characteristics Associated With Survival Among Patients Treated With Checkpoint Inhibitors for Advanced NonSmall Cell Lung Carcinoma: A Sys-tematic Review and Meta-analysis. JAMA Oncol 2018;4:210-6.

81. Jeanson A, Tomasini P, Souquet-Bressand M, et al. Efficacy of Immune Checkpoint Inhibitors in KRAS-Mutant NonSmall Cell Lung Cancer (NSCLC). J Thorac Oncol 2019;14:1095-101.

82. Passiglia F, Cappuzzo F, Alabiso O, et al. Efficacy of nivolumab in pre-treated non-small-cell lung cancer patients harbouring KRAS mutations. Br J Cancer 2019;120:57-62.

83. Amaral T, Sinnberg T, Meier F, et al. The mitogenactivated protein kinase pathway in melanoma part I Activation and primary resistance mechanisms to BRAF inhibition. Eur J Cancer 2017;73:85-92.

84. Cantwell-Dorris ER, O'Leary JJ, Sheils OM. BRAFV600E: implications for carcinogenesis and molecular therapy. Mol Cancer Ther 2011;10:385-94.

85. Paik PK, Arcila ME, Fara M, et al. Clinical characteristics of patients with lung adenocarcinomas harbor-ing BRAF mutations. J Clin Oncol 2011;29:2046-51.

86. Marchetti A, Felicioni L, Malatesta S, et al. Clinical features and outcome of patients with non-small-cell lung cancer harboring BRAF mutations. J Clin Oncol 2011;29:3574-9.

87. Cardarella S, Ogino A, Nishino M, et al. Clinical, pathologic, and biologic features associated with BRAF mutations in non-small cell lung cancer. Clin Cancer Res 2013;19:4532-40.

88. Ding X, Zhang Z, Jiang T, et al. Clinicopathologic characteristics and outcomes of Chinese patients with nonsmall-cell lung cancer and BRAF mutation. Cancer Med 2017;6:555-62.

89. Zaman A, Wu W, Bivona TG. Targeting Oncogenic BRAF: Past, Present, and Future. Cancers (Basel) 2019;11:1197.

90. Planchard D, Kim TM, Mazieres J, et al. Dabrafenib in patients with BRAF(V600E)-positive advanced non-smallcell lung cancer: a single-arm, multicentre, open-label, phase 2 trial. Lancet Oncol 2016;17:642-50.

91. Planchard D, Besse B, Kim TM, et al. Updated survival of patients (pts) with previously treated BRAF V600Emutant advanced non-small cell lung cancer (NSCLC) who received dabrafenib (D) or D + tra-metinib (T) in the phase II BRF113928 study. J Clin Oncol 2017;35:9075.

92. Hyman DM, Puzanov I, Subbiah V, et al. Vemurafenib in Multiple Nonmelanoma Cancers with BRAF V600 Mutations. N Engl J Med 2015;373:726-36.

93. Dudnik E, Peled N, Nechushtan H, et al. BRAF Mutant Lung Cancer: Programmed Death Ligand 1 Expression, Tumor Mutational Burden, Microsatellite Instability Status, and Response to Immune Check-Point Inhibitors. J Thorac Oncol 2018;13:1128-37.

94. Yoh K, Matsumoto S, Kunimasa K, et al. The efficacy of immune checkpoint inhibitors and PD-L1 sta-tus in patients with advanced non-small cell lung cancer harboring oncogenic driver alterations: Immu-nooncology biomarker study in LC-SCRUM-Japan. J Clin Oncol 2019;37:9046.

95. Guisier F, Dubos-Arvis C, Viñas F, et al. Efficacy and Safety of Anti-PD-1 Immunotherapy in Patients With Advanced NSCLC With BRAF, HER2, or MET Mutations or RET Translocation: GFPC 01-2018. J Thorac Oncol 2020;15:628-36.

96. Bergethon K, Shaw AT, Ou SH, et al. ROS1 rearrangements define a unique molecular class of lung cancers. J Clin Oncol 2012;30:863-70.

97. Sabari JK, Leonardi GC, Shu CA, et al. PD-L1 expression, tumor mutational burden, and response to immunotherapy in patients with MET exon 14 altered lung cancers. Ann Oncol 2018;29:2085-91.

98. Drilon A, Clark J, Weiss J, et al. OA12.02 Updated Antitumor Activity of Crizotinib in Patients with MET Exon 14-Altered Advanced Non-Small Cell Lung Cancer. J Thorac Oncol 2018;13:S348.

99. Wolf J, Seto T, Han JY, et al. Capmatinib (INC280) in MET $\Delta$ ex14-mutated advanced non-small cell lung cancer (NSCLC): Efficacy data from the phase II GEOMETRY 
mono-1 study. J Clin Oncol 2019;37:9004.

100. Paik PK, Veillon R, Cortot AB, et al. Phase II study of tepotinib in NSCLC patients with METex14 mu-tations. J Clin Oncol 2019;37:9005.

101.Lu S, Fang J, Cao L, et al. Abstract CT031: Preliminary efficacy and safety results of savolitinib treating patients with pulmonary sarcomatoid carcinoma (PSC) and other types of non-small cell lung cancer (NSCLC) harboring MET exon 14 skipping mutations. Cancer Res 2019;79:CT031-CT.

102. Kauffmann-Guerrero D, Tufman A, Kahnert K, et al. Response to Checkpoint Inhibition in Non-Small Cell Lung Cancer with Molecular Driver Alterations. Oncol Res Treat 2020;43:289-98.

Cite this article as: McLean L, Leal JL, Solomon BJ, John T. Immunotherapy in oncogene addicted non-small cell lung cancer. Transl Lung Cancer Res 2021;10(6):2736-2751. doi: $10.21037 /$ tlcr-20-772 